\title{
Single Image Super-Resolution Using Multi-Scale Deep Encoder-Decoder with Phase Congruency Edge Map Guidance
}

Heng Liu ${ }^{\mathrm{a}}$, Zilin $\mathrm{Fu}^{\mathrm{a}}$, Jungong $\mathrm{Han}^{\mathrm{b}}$, Ling Shao ${ }^{\mathrm{c}}$, Shudong Hou ${ }^{\mathrm{a}}$, Yuezhong $\mathrm{Chu}^{\mathrm{a}}$

${ }^{a}$ Anhui University of Technology, China, 243032

${ }^{b}$ Lancaster University, U.K., LA1 $4 Y W$

${ }^{c}$ University of East Anglia, U.K., NR4 7TJ

\begin{abstract}
This paper presents an end-to-end multi-scale deep encoder (convolution) and decoder (deconvolution) network for single image super-resolution (SISR) guided by phase congruency (PC) edge map. Our system starts by a single scale symmetrical encoder-decoder structure for SISR, which is extended to a multi-scale model by integrating wavelet multi-resolution analysis into our network. The new multi-scale deep learning system allows the low resolution (LR) input and its PC edge map to be combined so as to precisely predict the multi-scale super-resolved edge details with the guidance of the highresolution (HR) PC edge map. In this way, the proposed deep model takes both the reconstruction of image pixels' intensities and the recovery of multiscale edge details into consideration under the same framework. We evaluate the proposed model on benchmark datasets of different data scenarios, such as Set14 and BSD100 - natural images, Middlebury and New Tsukuba depth images. The evaluations based on both PSNR and visual perception reveal that the proposed model is superior to the state-of-the-art methods.
\end{abstract}

Keywords:

single image super-resolution, multi-scale deep model, deep

encoder-decoder, phase congruency edge map 


\section{Introduction}

SISR usually refers to reconstructing or recovering an HR image from an LR image without losing high frequency details or reducing the image quality, where the LR image is usually undergone a degradation process, such as geometric deformation, motion blurring and down sampling. Superresolving an LR image is basically an inverse process of the degradation model, with the goal to recover the missing high-frequency details in the original HR image, such as edges and textures. Obviously, the solution of such recovery is very ambiguous because there are many HR images that can produce the same LR image. Therefore, SISR is a highly ill-posed problem due to its non-unique solution.

In general, SISR can be implemented by three means, namely, the interpolation based methods, the reconstruction based methods and the learning based methods [41, 42, 3, 48, 47]. The simple and fast SR methods employ different discrete interpolations, such as linear, bilinear and bi-cubic interpolations, all of which rely on the smoothness assumptions. However, the smoothness assumption will result in jaggy and ringing effects due to the discontinuities in images. Reconstruction based SR methods, especially maximum a posteriori (MAP), actually model the image degradation process, which mainly focuses on how to get the forward observation model and how to achieve SR through the degradation model. In general, both interpolation and reconstruction based methods merely process the image signal at the pixel level. On the contrary, learning based methods, especially dictionary learning or sparse representation based ones [41, 42], pay more attention to the understanding of the image content and structure, for which the prior knowledge in relation to image data imposes the constraints on the data for a better reconstruction. Given the training dataset, learning based SISR methods intends to learn an implicit mapping function between the low resolution (LR) images and the high resolution (HR) images, which have received considerable attention in the past few years.

In recent years, deep learning, especially convolutional neural networks (CNNs), have achieved amazing success in handling various vision tasks, including image classification, object detection, image de-noising and so on. Due to the simplicity of an end-to-end training and the superior performance, have been applied into diverse vision challenges. Compared with traditional hand-designed feature extraction, CNNs can directly learn data

representations from raw training samples and detect data-driven features 
for specific tasks. In addition, unlike traditional learning based methods, CNN based methods are end-to-end and can be more capable of directly approximating mapping function between LR images and HR images, which can integrate intermedia processing into one pipeline handily. The seminal work of SISR based on convolutional neural network (SRCNN) is first proposed by Dong et al. [4], where an implicit LR to HR mapping is acquired via building a three-layer convolutional network and superior performance has been achieved. In such a model, image patch extraction, representation, non-linear mapping and image reconstruction, are sequentially implemented, which tend to simulate the processing procedure of sparse coding to generate HR images. Without considering the image prior, SRCNN model becomes a generic framework for image super resolution due to its lightweight structure and the end-to-end style. However, recent works [37, 6, 15] acknowledge that the deeper and more complex networks can lead to more superior SR performance, which significantly raises the difficulty of network training. In addition, the research works in $[21,43,38]$ argue that introducing image edge priors will either accelerate the training convergence [21] or help improve the reconstruction quality $[43,38]$. Nevertheless, issues such as what type of image edge priors suits better and what deep convolution network structure is appropriate to this task are not fully addressed in existing CNNs based works. Thus, the exploration of constructing a deeper and more complex network with favorable image edge priors integration needs to be carried out in depth.

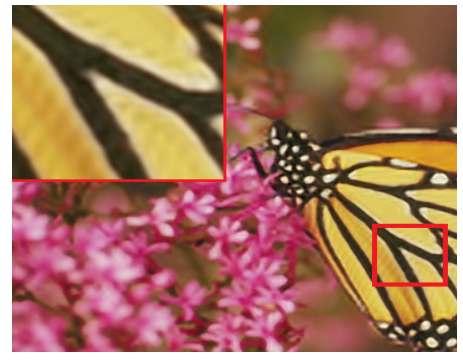

(a) SRCNN-L [5]

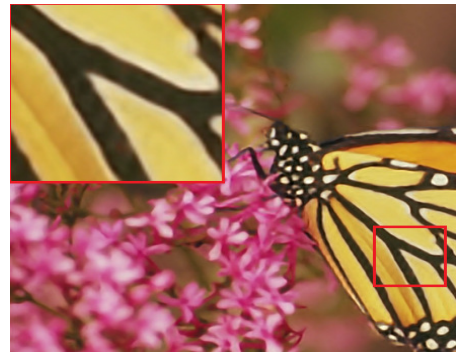

(b) MSDEPC (Proposed)

Figure 1: The edges in the super-resolved image produced by the proposed MSDEPC model (right) are much sharper than the ones produced by SRCNN-L [5] (left) (4×downsampling).

Other deep supervised models (including those in [20, 19]), especially fully convolutional networks (FCNs) $[22,40]$, are proposed recently for image semantic segmentation and object detection. Eliminating the fully connected 
layers, FCN is all composed of convolution and deconvolution operations, which are usually named as encoder and decoder, respectively. However, in the above FCNs, convolution is always followed by pooling while deconvolution is followed by un-pooling, thus directly applying FCNs for image restoration tasks may give rise to the loss of the image details. Moreover, most existing FCN works only consider the single scale convolution pipeline, which fails to take full use of multiple range context and image details for SISR. It should be pointed out that in all of the followings, without special statement, the items of encoder and decoder always refer to the operations of convolution and deconvolution.

In this work, for CNN based image SR, we want to investigate whether all kinds of image edge features are suitable and helpful, and how to construct an opportune deep model with the favorable of edge details. Considering the fact that multi-scale image contextual information is essential for the reconstruction of high-frequency image details, based on the network simulation of discrete wavelet multi-resolution analysis, we propose a multi-scale deep encoder-decoder structure for SISR. More specifically, we construct a threescale encoder-decoder deeper network by cascading three convolution and deconvolution layers with varying length sizes. At each scale, deep encoderdecoder does not involve any pooling and un-pooling operations in order to avoid image details leaking during the image recovering. Then, motivated by the observation that phase congruency (PC) edge map [17], a kind of structural edge features of an image, is invariant against different scales subsampling, we manage to introduce the phase congruency edge map to guide the prediction of the edge features in our multi-scale model. By doing so, the proposed multi-scale deep SR model becomes capable of recovering the edge details alongside reconstructing the image intensities at different scales, in which the edge loss and the pixels' value loss are combined to jointly supervise the training. An example of a super-resolved image through the proposed model from $4 \times$ down-sampling is illustrated and compared to SRCNN-L [5] in Fig. 1. Our proposed SISR deep model (abbreviated as MSDEPC) is illustrated in Fig. 2.

In summary, the contributions of our work are three-fold:

- By using network operations to simulate wavelet multi- resolution analysis, we design a novel end-to-end multi-scale deep encoder-decoder with edge map guidance for SISR. In this model, image data and the corresponding edge maps are simultaneously fed into the pipeline. Along the multiple streams, convolution-deconvolution responses with different scales are concatenated 


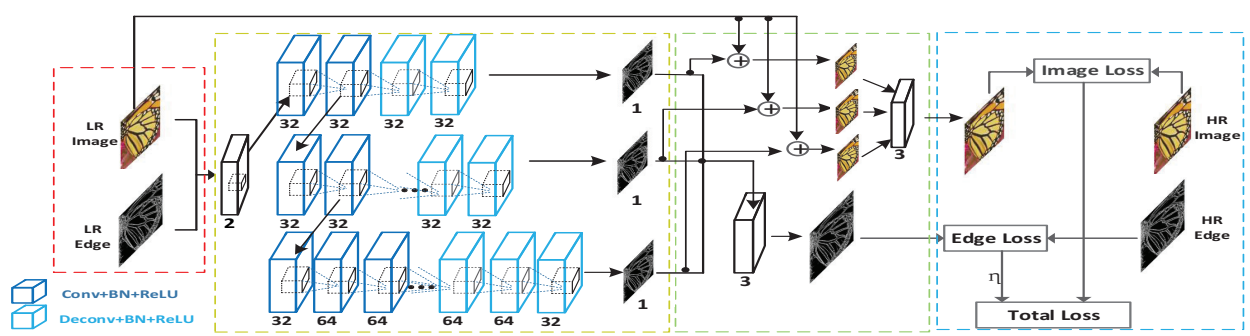

Figure 2: The proposed MSDEPC model: joint input of LR image and edge (red), multiscale encoder-decoder learning (yellow), multi-scale HR image and edge prediction (green), and the total loss (blue).

to generate the final reconstructed image.

- We integrate the PC edge prior into the deep network and balance the image intensity loss and the edge loss to jointly supervise the training. The comparisons and the experimental results show that the PC edge map is more suitable for SISR, compared to other types of edges, e.g., Canny edges and Sobel edges, in terms of the reconstruction quality.

- We verify and evaluate the proposed model on widely recognized public datasets, and also on completely different data from training images, e.g., depth images. We also compare the variants of our model and discuss the impact when taking different local structures of the network, which will be constructive and helpful for future SISR research.

The rest of this paper is organized as follows. In Section 2, the related works for SISR are reviewed in detail. In Section 3, the architecture and the constructing principle of the proposed deep model are described. Experimental results and discussions are provided in Section 4. Finally, Section 5 makes a brief conclusion and outlines the future work.

\section{Related Work}

\subsection{Image Priors based Super-Resolution}

As known, for image recovery tasks, image priors play an important role in regularizing the optimization process or imposing a constraint for a fast solution. So far, some image priors have been successfully applied for SISR, such as sparse prior [41, 42], exemplar prior [35, 46], and self-similarity prior $[11,49]$.

Yang et al. [41, 42] introduced a sparse prior based method by learning a coupled dictionary of HR and LR images, in which the representation coefficients of the HR patches and the corresponding LR patches are assumed 
to be the same. Based on exemplar prior, Timofe et al. [35] proposed an anchored neighbourhood regression based approach, where the anchors refer to the learned dictionary atoms. Alternatively, Zhang et al. [46] utilized a mixture of experts (MoE) method to jointly learn the feature space partition and local regression models. Some recent studies in $[11,49]$ reveal that local image structure tends to occur within and across different image scales. Due to such self-similarity prior, image SR can be solved by using self-similar examples instead of the external data. On top of it, the internal patch search space of self-similarity methods can even be expandable if allowing geometric variations [11]. In addition, $\mathrm{Gu}$ et al. [6] regarded sparse prior based image $\mathrm{SR}$ as a filtering process. To address the inconsistency problem of pixels in the overlapped blocks, they raised a convolutional sparse coding based SR. However, this method, especially the training part, is rather expensive in the sense that three groups of parameters that need to be learned: LR filters, mapping functions and HR filters.

,some image priors have been recently introduced into the CNNs for SISR $[37,21]$, which will be elaborated below.

\subsection{CNN based Image Super-Resolution}

Benefiting from the powerful non-linear mapping, CNN based image SR, including the pioneer SRCNN [5, 4] and the very recent works $[8,7,28]$, can improve the performance dramatically compared with the traditional methods. One main weak of SRCNN is that the model actually is not deep enough and is trained without the prior knowledge considered, thereby leading to a very slow convergence speed. In [37], Wang et al. presented a compositional model combining sparse prior and a deep network, which demonstrates an efficient training/performance trade-off for SISR. Considering that directly training an SRCNN model takes too long to converge, Liang et al. [21] introduced Sobel edge detection so as to capture gradient information to accelerate the training convergence. In fact, the method does reduce the training time but the resultant reconstruction enhancement is limited. Again exploiting Sobel edge features, Yang et al. [43] described a recurrent residual learning method for SISR. However, in principle, Sobel edge features only acquire image magnitude step-jump discontinuities in both horizontal and vertical directions, but cannot preserve accurate and stable image edge details, especially when applying sub-samplings at different scales.

In addition to image priors, recent evidences [34, 9] have revealed that the network depth is of crucial importance and an appropriate deep structure 
can usually lead to a remarkable performance improvement. For example, Kim et al. [15] take twenty convolution layers with residual connected to construct SR network which accomplishes a significant improvement in SR reconstruction accuracy. Aiming to improve SISR, Ledig et al. [18] proposed to utilize a novel network structure - generative adversarial network (SRGAN) to produce photo-realistic SR images. The SRGAN network consists of two distinct sub-networks and adopts an extended version of the perception loss [14] for training, being defined based on the feature maps from the VGG network [34]. In spite of its surprisingly good performance, the latest evidence [27] suggests that the generated super-resolved image will be likely to contain some checkerboard artifacts. Moreover, in an effort to achieve real time SISR, Shi et al. [33] claim that directly learning upscaling filters through a network can improve the reconstruction performance both in accuracy and speed. Actually, the proposed sub-pixel convolution layer is almost equivalent to the deconvolution layer but it requires more convolution filters to produce enough feature maps.

\subsection{Deep Encoder and Decoder}

Abandoning fully connected layers, the fully convolutional networks (FCNs) containing both convolution and deconvolution layers have been applied to semantic segmentation [22] and object detection [40], where a convolutional layer serves as an encoder (feature extraction and representation) while a deconvolutional one acts as a decoder (reconstruction). Inspired by the success of these works, such encoder-decoder structure naturally appeals for application in image super-resolution. However, due to the possibility of discarding useful image details, all pooling and un-pooling layers which usually exist in FCN should be removed. Such proposed deep encoder-decoder symmetrical network is shown in Fig. 3. In addition, with the gradual realization of the nature of hierarchical learning in convolution networks, multi-scale learning appears in some recent works, including edge detection [39], skeleton extraction [32] and image dehazing [29]. The common characteristics of these multi-scale works takes different length convolution branches or equivalently different sizes filters to achieve the different sizes of receptive fields so as to extract the image features at different scales. Based on such observation and taking the principle of wavelet multi-resolution analysis to guide multi-scale network construction, the single scale encoder-decoder symmetrical structure can be naturally extended to multiple scales deep model (see Fig. 2), which will be elaborated in the following sections. 


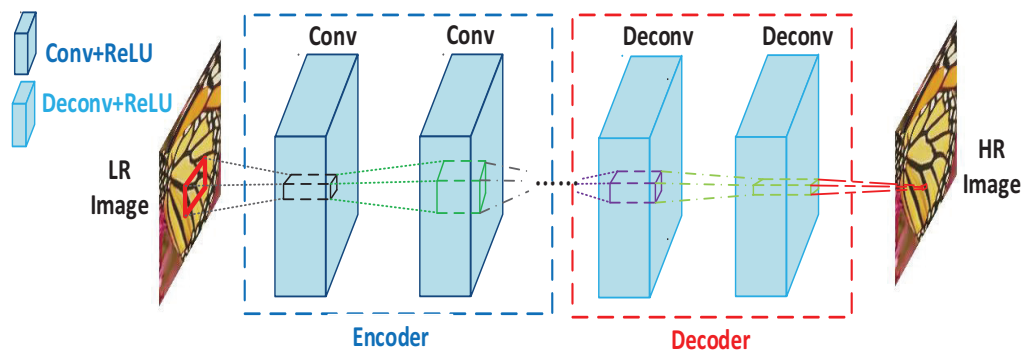

Figure 3: Single scale deep symmetrical encoder-decoder: the network only consists of convolutional and deconvolutional layers; PReLU layer follows after convolution or deconvolution operation.

\section{Multi-scale Deep Encoder-Decoder Learning for Image SR}

\subsection{Design Basis for Multi-scale Encoder-Decoder}

Designing a multi-scale deep encoder-decoder gains two main benefits: 1) multi-scale features can be easily extracted from the input image by multiple scales encoders and the SR image under different scales can be reconstructed through different scales decoders; 2) it is an end-to-end system, implying that it is convenient to adjust the network itself and observe the effects. However, due to the absence of previous references or reports, how to design a multi-scale encoder-decoder network for SISR becomes a new challenge. Fortunately, since SISR can be treated as a signal reconstruction task, we may take wavelet analysis [24], a well-known method for signal decomposition and reconstruction, to guide the network construction of multi-scale deep encoder-decoder. Actually, in this work, we adopt the network simulation strategy and implement the multi-scale analysis and reconstruction by the corresponding network operations, such as convolution, deconvolution, concation and summation.

Based on the wavelet multi-resolution analysis (MRA) [24], for an image $f(x)$ in $L^{2}$ space $R$, it can be represented as:

$$
f(x)=\sum_{k \in Z}^{N} a_{k}^{j_{0}} \phi_{k}^{j_{0}}(x)+\sum_{j=j_{0}}^{J} \sum_{k} b_{k}^{j} \psi_{k}^{j}(x),
$$

where $j$ is the scale varying from $j_{0}$ to $J, k$ is the index of basis function, and $\left\{a_{k}^{j_{0}}\right\},\left\{b_{k}^{j}\right\}$ are coefficients attached to the approximation (scale) function $\phi(x)$ and the detail (wavelet) function $\psi(x)$, respectively. Specifically and in short, the image $f(x)$ can be viewed as consisting of two components 
(see Eq. (1)): the approximation (the first item, low frequency component) and the details (the second item, high frequency components). That is if varying the scale $\mathrm{j}$ from zero to certain scale, $f(x)$ can be represented as the weighted summation of a series of components or (sub-bands) at different scales, which contains a low frequency approximation and several or numerable high frequency details. From deep learning point of view, Eq. (1) on the whole may be treated as the combination of deconvolution (reconstruction) operations at multiple scales. In the equation, the approximation coefficients $a_{k}^{j}$ and the detail coefficients $b_{k}^{j}$ are the projections of image $f(x)$ to different approximation subspaces or detail subspaces at different scale $j$. Actually, $a_{k}^{j}$ and $b_{k}^{j}$ can be calculated as:

$$
\begin{aligned}
& a_{k}^{j}=\left\langle f(x), \phi_{k}^{j}(x)\right\rangle=\sum_{i} p_{i k}^{j} f_{i} \\
& b_{k}^{j}=\left\langle f(x), \psi_{k}^{j}(x)\right\rangle=\sum_{i} q_{i k}^{j} f_{i}
\end{aligned}
$$

Here, assuming the image $f(x)$ is discretized as $f=\left\{f_{1}, f_{2}, \cdots, f_{i}, \cdots\right\}$, the scale function $\phi_{k}^{j}$ is relaxed to $\left\{p_{1 k}^{j}, p_{2 k}^{j}, \cdots, p_{i k}^{j}, \cdots\right\}$, and the wavelet function $\psi_{k}^{j}$ is relaxed to $\left\{q_{1 k}^{j}, q_{2 k}^{j}, \cdots, q_{i k}^{j}, \cdots\right\}$. According to convolutional hierarchical feature learning in [44], if regarding the weights $p_{i k}^{j}$ and $q_{i k}^{j}$ as the kernels of the convolution layers at certain scale $j$ and treating the inner projection as the procedure of feature encoding, Eq. (2) will be realized by one scale encoding (convolution) on the image $f(x)$. Accordingly, $a_{k}^{j}$ and $b_{k}^{j}$ will become the feature map outputs of these encoders at certain scale. Naturally, if also introducing the decoder's weights $\tilde{p}_{i k}^{j}$ and $\tilde{q}_{i k}^{j}$ as the kernels of the deconvolution layers, then based on Eq.(1) the reconstructed image $\tilde{f}(x)$ can be obtained by multi-scale decoding (deconvolution) as:

$$
\tilde{f}(x)=\sum_{j} \sum_{k} \sum_{i} \tilde{p}_{i k}^{j} a_{k}^{j}+\sum_{j} \sum_{k} \sum_{i} \tilde{q}_{i k}^{j} b_{k}^{j}
$$

In addition, according to [34], the effect of cascading a serial of convolution layers is equivalent to the convolution of a particular scale. In summary, cascading the convolution layers and the deconvolution layers sequentially with different lengths enables us to construct a multi-scale deep encoderdecoder for SISR. 


\subsection{Multi-scale Encoder-Decoder Learning}

Obviously, by cascading the convolution and deconvolution with different lengths, the encoding and decoding of image $f(x)$ can be carried out continuously from coarse scale (short stream) to fine scale (long stream). Assuming the network stream at each scale, denoted as $\tilde{f}_{j}$, represents an approximation of $f(x)$. Thus, according to Eq. (3), if we take a summation function $s$ adding up all encoder-decoder streams, the multi-scale encoder-decoder reconstruction $\tilde{f}(x)$ can be easily acquired as:

$$
\tilde{f}(x)=s\left(\tilde{f}_{1}, \tilde{f}_{2}, \cdots, \tilde{f}_{j}, \cdots\right)
$$

Therefore, through such multi-scale expansion, the primary content and the details of an HR image can be gradually recovered. Particularly, if we regard the LR image also as an approximation of HR image $f$ and input it to the network, the super-resolved image $\tilde{f}$ can be generated from the multi-scale encoder-decoder structure by replacing the summation function $s$ in Eq. (4) with the network summation operation.

The optimization target of multi-scale encoder-decoder learning can be regarded as:

$$
\tilde{f}=\arg \min _{f}\left(\sum_{j}\left\|F_{j}^{a}\left(y, \Theta_{j}\right)-f_{j}^{a}\right\|_{2}^{2}+\sum_{j}\left\|F_{j}^{b}\left(y, \Theta_{j}\right)-f_{j}^{b}\right\|_{2}^{2}\right),
$$

where $f$ and $y$ represent the HR image and the corresponding LR image, and $F(\cdot)$ denotes the network reconstruction function. $\Theta$ is the learned parameter of the network and symbols $j, a, b$ indicate a specific scale, a low frequency approximation component and a high frequency component, separately. By taking into account the components of different scales simultaneously, multiscale encoder-decoder learning will overcome the deficiency of only considering the similarity of $L^{2}$-norm energy (mainly concentrated in low frequency components) whilst ignoring the recovery of the structural details (in high frequency components).

Given a set of LR and HR image pairs $\left\{f_{i}, y_{i}\right\}_{i=1}^{N}$ and assuming the approximation and detail components of HR image $f_{i}$ at multiple scales can be obtained, then according to Eq. (5), the loss function of the proposed multi-scale encoder and decoder can be denoted as:

$$
\text { Loss }=\sum_{j} \lambda_{j} \sum_{i=1}^{N}\left\|F_{j}^{a}\left(y_{i}, \Theta_{j}\right)-f_{j, i}^{a}\right\|^{2}+\sum_{j} \beta_{j} \sum_{i=1}^{N}\left\|F_{j}^{b}\left(y_{i}, \Theta_{j}\right)-f_{j, i}^{b}\right\|^{2},
$$


where $\lambda$ and $\beta$ are regulation coefficients for every loss term. All other symbols are the same as those in Eq. (5). However, to facilitate training and simplify regulation, the loss function can be relaxed to Eq. (8) by: 1) synthesizing multiple components of different scales to two components (low frequency approximation and high frequency detail, Eq. (7)); 2) replacing the approximation component with the input LR image and specifying the detail component as one special type of it - PC edge map.

$$
\begin{aligned}
& \operatorname{Loss}(\Theta) \approx \lambda \sum_{i=1}^{N}\left\|F^{a}\left(y_{i}, \Theta\right)-f_{i}^{a}\right\|^{2}+\beta \sum_{i=1}^{N}\left\|F^{b}\left(y_{i}, \Theta\right)-f_{i}^{b}\right\|^{2}, \\
& \operatorname{Loss}(\Theta) \approx \sum_{i=1}^{N}\left\|F\left(y_{i}, \Theta\right)-f_{i}\right\|^{2}+\eta \sum_{i=1}^{N}\left\|F\left(L e_{i}, \Theta\right)-H e_{i}\right\|^{2},
\end{aligned}
$$

where $\eta$ can be regarded as a trade-off, regulating the reconstruction focus of the energy approximation term (the first term, low frequency component) and the edge similarity term (the second term, high frequency details ). More discussions on the effect of each term in the loss function can be referred to the first part of Section 4.4. $L e_{i}$ and $H e_{i}$ denote the $i^{\text {th }}$ extracted LR and $\mathrm{HR}$ edges using the $\mathrm{PC}$ edge map operator, separately. $F\left(L e_{i}, \Theta\right)$ represents the prediction of the edge map of the super-resolved image.

\subsection{Edge Map Guidance}

If the observed LR image $y$ is regarded as the low-frequency component of the HR image, then the high frequency components are just the details of the image, such as textures, edges or corners. In other words, recovering image details becomes the most pivotal requirement for SISR. This motivates us to use the image details to guide SR image reconstruction rather than using the pixels' intensity values only. With respect to the proposed deep model, the guidance provided by image details is composed of two aspects: taking the HR image details for network supervision and integrating the corresponding LR image details with itself as the network input.

In fact, edges, textures, corners and other kinds of image details are different high-order statistics and they contain certain structural features of the image, among which edges represent one-order magnitudes varying and are most informative. However, directly calculating magnitude variations (such as Canny or Sobel operators) as edges will work well only on the step type. When applying sub-sampling at different scales, they will encounter 


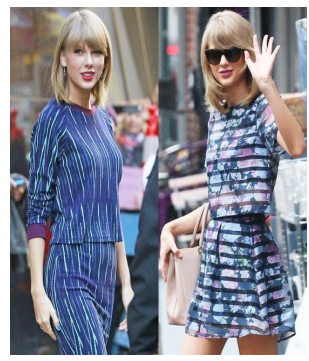

(a) Original

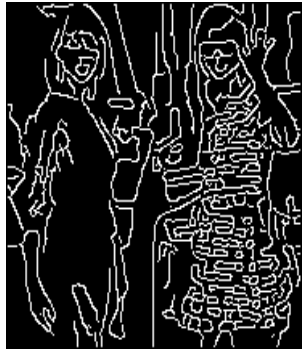

(e) Canny edge of $(f)$ $3 \times \mathrm{DS}$

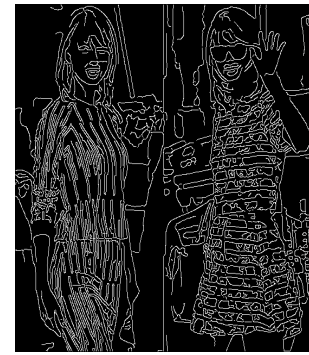

(b) Canny edge
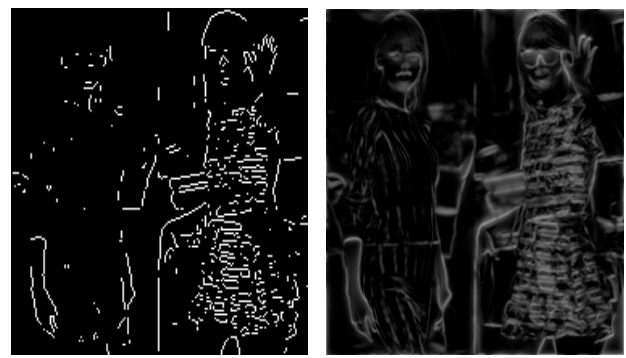

$3 \times \mathrm{DS}$

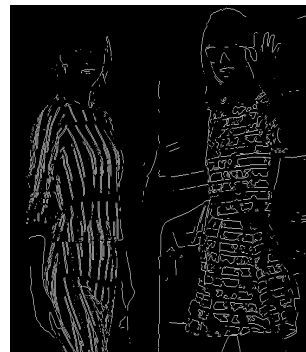

(c) Sobel edge

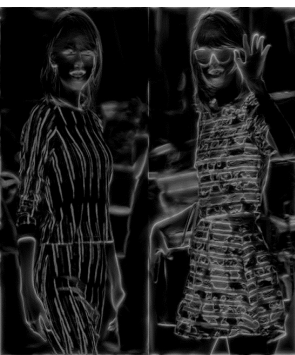

(d) PC edge map

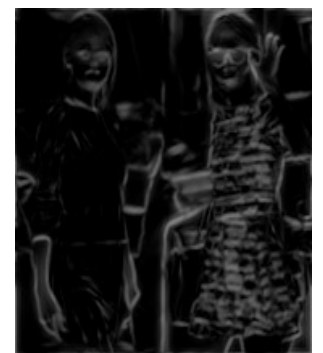

h) PC edge map of $3 \times \mathrm{DS}$

Figure 4: PC edge map vs. Canny or Sobel edge when down-sampling (DS) with different scales.

the problem that the edge location will not be consistent or will even be completely different.

In view of this, Kovesi in [16] argued that the Fourier components of a signal are all in phase at the point of the step in the square wave, and at the peaks and troughs of the triangular wave. This property, named phase congruency (PC), is stable over scale and intensity, which can be measured as the following if at a location $x$ :

$$
P C(x)=\frac{W(x) \inf (|E(x)|-T)}{\sum A(x)+\varepsilon},
$$

where $A(x)$ is the amplitude, $W(x)$ is a weighting function for frequency spread, $E(x)$ is local energy, $\varepsilon$ is small constant to avoid division by 0 , and $T$ is noise compensation. Moreover, phase congruency (PC) edge map [17], acquired by Log-Gabor multi-scale analysis on the PC map [16], is a kind of image edge feature map in which the phase of certain edges does not change even at different scales. That is to say, the PC edge map provides robust edge details across multi-scale sub-samplings. In view of this unique characteristic, $\mathrm{PC}$ edge map is extracted and exploited in our multi-scale encoder-decoder 
network to improve SISR by jointly supervising the training. More details concerning the PC edge map as well as its extraction may be found in [17]. The comparisons between the PC edge map and the traditional edge features are illustrated in Fig. 4.

We can take the stability and the consistency of the features across different scales to measure the performance difference of the edge feature extraction methods. As can be seen from Fig. 4, the traditional edge features (Canny or Sobel) of $3 \times 3$ down-sampling are obviously distinct from the original traditional edge features (see (e) to (b) and (f) to (c)). Compared to the original edge features, they lose many structural details and introduce some artifacts. In other words, the traditional edge features are not robust when image down-sampling occurs. Whereas PC edge maps across different scales are basically consistent and never produce any artifacts (see (h) to (g) and (d)). Therefore, the PC edge map is superior to the traditional edge features in terms of the stability and consistency at different scales.

\subsection{Model Architecture}

The architecture of the proposed model can be divided into four algorithmic steps (see Fig. 2). The specific configurations of all convolution and deconvolution layers can be found in Table 1.

In the first step, two components are integrated as input and fed into the network, that is, an LR image with its corresponding PC edge map. This can also be interpreted as we integrate the two components to learn.

In the second step, the joint input is sent to a multi-scale network to fuse learned multi-scale features. In our model, we use a three-scale encoderdecoder symmetrical network in order to obtain better image reconstruction performance. Next, the multiple streams are connected by side outputs similar to the connections in works $[39,32]$. Here, relatively fewer filters (e.g. 32 ) and smaller kernel sizes (e.g. $3 \times 3$ ) are adopted in the convolutional layers because we want to reduce the computation load of the network and we believe that connecting convolution layers through cascading can simulate any sizes of receptive field [34].

In the third step, the edge maps of three scales are firstly estimated by convolution, and afterwards they are synthesized to get the final estimation for the super-resolved edge. The estimated edge map then will be piped into the fourth step for edge feature loss computation. In addition, in the third step, since the input LR image can be regarded as the low frequency component of the HR image, it can be directly added with the estimated edge maps 
(see three direct links from the input to the summation units) to get three reconstructed images at different scales. Furthermore, the reconstructed ones are also integrated to get the final super-resolved image.

At last, in the fourth part, the image intensity loss and the edge map loss are combined with the weight $\eta$ for the supervised training.

In order to alleviate the difficulty in training convergence, PReLU activation function [10] with batch normalization layer [12] (BN) is added into our architecture. In practice, we find that such two tricks can also improve the reconstruction quality.

Table 1: The configuration of three scales encoder-decoder streams (4 layers, 8 layers and 12 layers): conv3 and deconv3 stand for convolution and deconvolution layers with kernel size $3 \times 3$ and stride $=1 ; 32$ and 64 are the numbers of filters.

\begin{tabular}{rcc}
\hline 4 Layers & 8 Layers & 12 Layers \\
\hline$($ conv3-32 $) \times 2$ & $($ conv3-32 $) \times 2$ & $($ conv3-32 $) \times 2$ \\
& $($ conv3-32 $) \times 2$ & $($ conv3-32 $) \times 2$ \\
& & $($ conv3-64 $) \times 2$ \\
& & $($ deconv3-64 $) \times 2$ \\
$($ deconv3-32 $) \times 2$ & $($ deconv3-32 $) \times 4$ & $($ deconv3-32 $) \times 4$ \\
\hline
\end{tabular}

\subsection{Computation Complexity}

From the computation point of view, a deep learning model, especially a convolutional neural network, needs to perform forward computation (making the input convolution and the output estimation) and backward propagation (computing the gradient and making the gradient convolution). Obviously, the computation of the whole deep network depends on the computation of each layer. The most complex (the most time-consuming) computation of each layer is the convolution, the time complexity of which dominantly depends on the parameters of the layer and the size of the input data to it. Since the proposed multi-scale deep encoder-decoder is a kind of fully convolutional network model, its computational complexity can actually be deduced from the complexity of a single convolution layer.

For any convolution layer, assuming the number of input channel is $C_{i n}$, the number of output channel is $C_{\text {out }}$, the size of feature map (output) is $M$, and the size of convolution kernel is $K$, then the time complexity of the convolution layer is:

$$
\text { Time } \sim O\left(M^{2} \cdot K^{2} \cdot C_{\text {in }} \cdot C_{\text {out }}\right)
$$


Thus, for the proposed multi-scale encoder-decoder, assuming there are $S$ scales, the total number of all convolution layers in each scale network (the depth) is $D_{s}$, the numbers of the input channel and the output channel of the $l^{\text {th }}$ convolution layer in each scale branch are $C_{l-1}$ and $C_{l}$, respectively, then the time complexity of the multi-scale model is:

$$
\text { Time } \sim O\left(\sum_{s=1}^{S} \sum_{l=1}^{D_{s}} M_{l}^{2} \cdot K_{l}^{2} \cdot C_{l-1} \cdot C_{l}\right)
$$

The space complexity of the proposed method depends on the parameters of the model, which can be formulated as:

$$
\text { Space } \sim O\left(\sum_{s=1}^{S} \sum_{l=1}^{D_{s}} K_{l}^{2} \cdot C_{l-1} \cdot C_{l}\right)
$$

\section{Experiments and Analysis}

\subsection{Datasets and Evaluation Measures}

We perform experiments and compare the algorithm performance on three widely acknowledged test data sets: Set5 [2], Set14 [45] and BSD100 [25]. For a fair comparison, our model is firstly trained with 91 images [35] which were extensively used in the previous works. Then, the entire framework is re-trained from scratch with 50,000 images collected from ImageNet [30] similar to $[5,18,33]$. Regarding the quality measurement of the reconstructed images, despite some recent no-reference metric works, such as [23], we still use the well-known PSNR [dB] and SSIM [36] metrics. One specific network is trained per super-resolution factor.

\subsection{Training Details}

For the 91-image training dataset, each image is augmented to 32 images by flipping and rotation, thus yielding a training set of 2730 images and a validation set of 182 images. Similar to the previous works, the network is only trained on the luminance component of images. For color images, the other two chrominance channels are simply up-scaled using a bi-cubic interpolation for the final output. Training images are cropped into small overlapped patches with a size of $50 \times 50$ pixels (larger than $33 \times 33$ in [5]) and a stride of 14 . The cropped ground truth patches were used as the highresolution patches, namely the target in our experiments. The corresponding 
low-resolution pairs are acquired by imposing the bi-cubic interpolation twice (same to the works $[4,5]$ ) on the ground truth, and the PC edge pairs are extracted with multiple scales by Log-Gabor filter banks at the same time.

In our framework, we follow the suggestions from He et al. [10, 9] to initialize the weights. Whilst training the framework, we initially set the learning rate to 0.01 and enable gradient clipping (GC). In our experiments, we clip the gradient to 10 first, and then change it to 1 when the loss value plateaus. Momentum and weight decay parameters are set to 0.9 and 0.0001 , respectively. The edge loss coefficient $\eta$ is a hyper parameter which can be determined by cross-validation with multiple folds or by the random search approach [1]. In practice, we set it to be 1 at the beginning and then manually adjusted it to 0.1, emphasizing the impact of image intensity reconstruction once the gradient becomes relatively small. The whole deep network training is implemented using the Caffe package [13] with one TITANT $\mathrm{X}$ GPU. The training loss convergence curve of $4 \times$ down-scaling SISR is shown in Fig. 5. The source code and the model can be downloaded at https://github.com/hengliusky/Muti-scale_SuperResolution. As for the ImageNet dataset training, all settings are the same as the 91-image dataset.

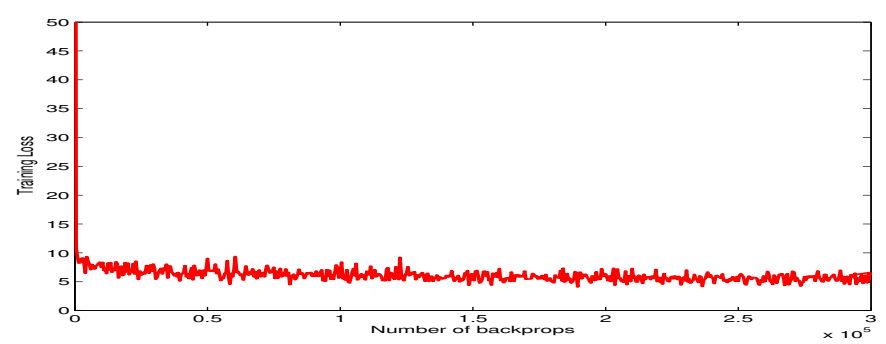

Figure 5: $4 \times$ down-scaling training convergence curve.

\subsection{Variant Models}

As mentioned above, our system consists of four parts. Modifying/changing any of them with other structure will bring about the variants of our model. A special variant is to replace the multiple scales parts (yellow and green parts in Fig. 2) with single scale structure while maintaining PC edge map. We name such special varaint as MSDEPC-V1. In addition, based on MSDEPC, other trivial variants include substituting the PC edge map with other image edge features, such as Canny edges and PC map [16] (containing more types of features than the PC edge map) while keeping the model's 
overall structure unchanged. We will train these variant models and compare their reconstruction performance in the following to illustrate whether they are suitable or not for our multi-scale encoder-decoder based SISR.

\subsection{Analysis and Comparisons}

Impact Analysis of The Terms in Loss Function. The terms in loss function, see Eq.8, are the energy approximation term (the first term) and the edge similarity term (the second term), respectively. To clarify the impact of each of these two terms for SISR, we separately utilize each term to supervise the network learning and then evaluate the corresponding reconstruction results. Here, all training settings and parameters are exactly the same as those of original $4 \times \mathrm{SR}$ model. The convergence curve and some reconstruction results of the edge similarity supervision are illustrated in Fig. 6 whereas the corresponding ones of the image energy approximation term are shown in Fig. 7.
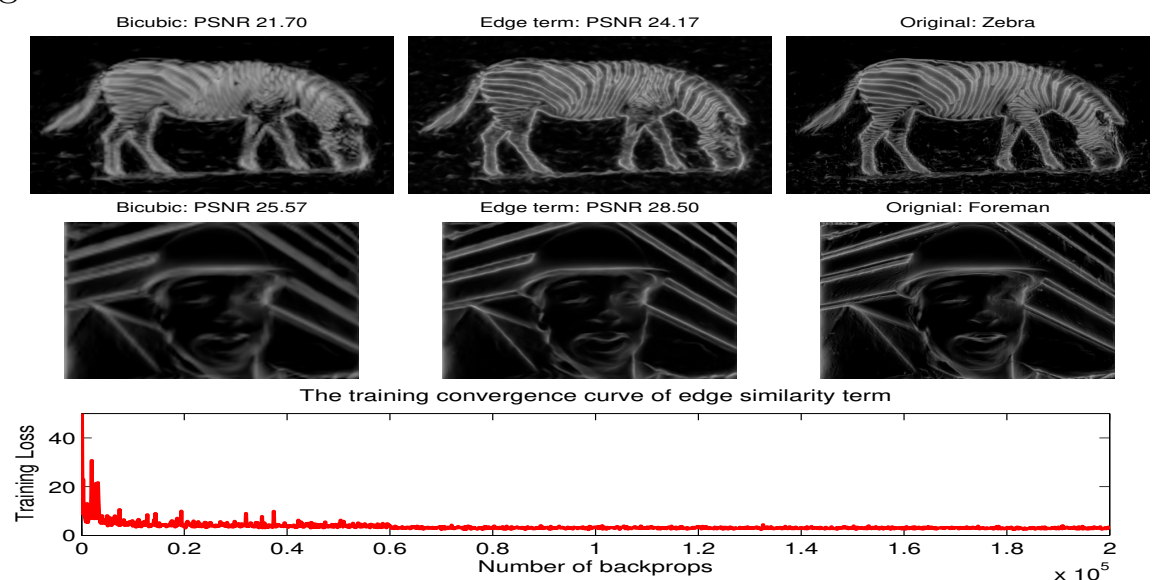

Figure 6: The effect of edge similarity supervision: $4 \times$ down-scaling training convergence curve (the bottom row); some reconstructed edges (the middle column of upper two rows).

It is clear that each term (edge similarity term and energy approximation term) of the loss function does work for SISR. Also, we can get that the edge similarity term makes the network learning to converge more stable and faster than the energy approximation term. In addition, comparing the objective metrics of the reconstructed results in Fig. 7 with those values of the proposed model (MSDEPC) in Fig. 9 (from the same label images, 'Zebar' and 'Foreman'), it is obvious that the edge similarity term does play a significant role in enhancing the performance of SISR: improve the PSNRs $(\mathrm{db})$ from 26.40 to 26.54 ('Zebra') and from 32.53 to 32.90 ('Foreman'), respectively. 

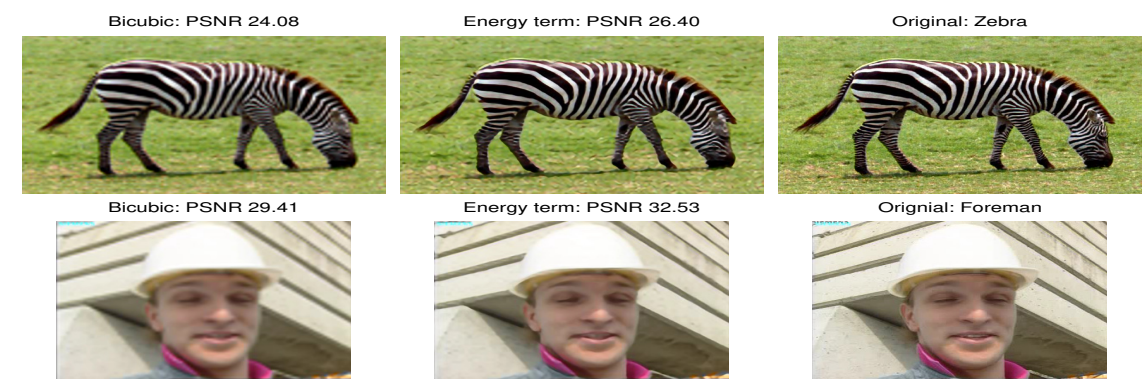

The training convergence curve of energy approximation term

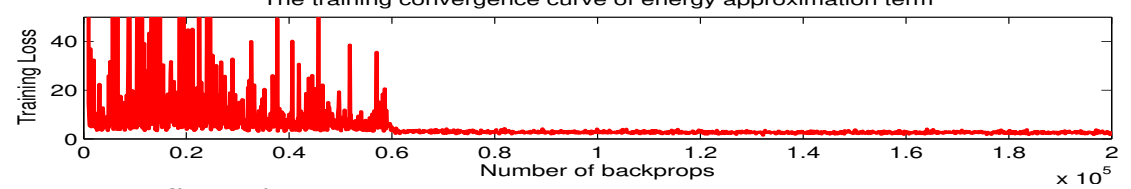

Figure 7: The effect of image energy approximation learning: $4 \times$ down-scaling training convergence curve (the bottom row); some reconstructed images (the middle column of upper two rows).

Guidance Effectiveness of PC Edge Map. In order to investigate the guidance effectiveness of PC edge map for SISR, in Table 2, we compare the reconstruction performance of those variants which take the PC map [16] or Canny edge features to replace the original PC edge with no structure changed. For ease of explanation, such two variants are denoted as MSDEPC-V2 and MSDEPC-V3, respectively. Meanwhile, MSDEPC-V1 mentioned above is also included in Table 2. Additionally, for a more clear understanding of the role of the PC edge map input, in the table we also list the performance of the deep encoder-decoder symmetrical network (abbreviated as DEDSN), which has no any edge input. The structure of DEDSN is shown in Fig. 3. Here, for convenience only the single scale is considered. Note that the configurations and the parameters of DEDSN are exactly the same as that of MSDEVP-1, except that it only uses LR images as input.

As a result, we have in total five deep frameworks involved in the comparison, alongside the baseline model SRCNN. Testing those variants effectively verifies the proposed contributions, which are the multi-scale deep encoderdecoder learning framework and the involvement of the PC edge map in our model training. It should be noted that in such comparisons all variants are trained based on the 91-image dataset, retaining the same settings of the original MSDEPC model.

According to Table 2, it is clear that among various types of image details, edge features do play an important role in the multi-scale encoder-decoder 
Table 2: The SR performance comparisons of the variants in terms of the averaged PSNR (dB) and SSIM [36] (upper and lower numbers, respectively). Best results are indicated in Bold.

\begin{tabular}{ccccccc}
\hline \multirow{2}{*}{ Dataset } & \multicolumn{2}{c}{ Set5 } & \multicolumn{2}{c}{ Set14 } & \multicolumn{2}{c}{ BSD100 } \\
\cline { 2 - 7 } & $\times 3$ & $\times 4$ & $\times 2$ & $\times 3$ & $\times 2$ & $\times 4$ \\
SRCNN[4] & 32.37 & 30.03 & 32.18 & 29.00 & 31.11 & 26.70 \\
& 0.9033 & 0.8530 & 0.9017 & 0.8145 & 0.8835 & 0.7078 \\
MSDEPC-V1 & 32.90 & 30.78 & 32.74 & 29.34 & 31.58 & 26.90 \\
& 0.9125 & 0.8745 & 0.9088 & 0.8196 & 0.8920 & 0.7135 \\
MSDEPC-V2 & 32.71 & 30.45 & 32.47 & 29.05 & 31.29 & 26.73 \\
& 0.9095 & 0.8696 & 0.9040 & 0.8098 & 0.8851 & 0.7074 \\
MSDEPC-V3 & 31.47 & 29.67 & 31.94 & 28.70 & 30.88 & 26.45 \\
& 0.8904 & 0.8506 & 0.8981 & 0.8066 & 0.8807 & 0.7011 \\
DEDSN & 32.75 & 30.53 & 32.42 & 29.10 & 31.41 & 26.78 \\
& 0.9105 & 0.8675 & 0.9031 & 0.8094 & 0.8869 & 0.7090 \\
MSDEPC & $\mathbf{3 3 . 3 7}$ & $\mathbf{3 1 . 0 5}$ & $\mathbf{3 2 . 9 4}$ & $\mathbf{2 9 . 6 2}$ & $\mathbf{3 1 . 6 4}$ & $\mathbf{2 7 . 1 0}$ \\
& $\mathbf{0 . 9 1 8 4}$ & $\mathbf{0 . 8 7 9 7}$ & $\mathbf{0 . 9 1 1 1}$ & $\mathbf{0 . 8 2 7 9}$ & $\mathbf{0 . 8 9 6 1}$ & $\mathbf{0 . 7 1 9 3}$ \\
\hline
\end{tabular}

model to improve the super-resolution quality. This conclusion can also be confirmed even if we compare DEDSN and MSDEPC-V1 separately. We will find that the former (without edge input) is much worse than the latter (with edge input) in terms of the performance. However, not all types of edge features are suitable for doing so, at least for our multi-scale model. For example, Canny edge features, introduced in the variant model - MSDEPC$\mathrm{V} 3$ is inappropriate for SISR in view of the lower PSNR and SSIM.

The fact that the framework guided by PC map (MSDEPC-V2) performs worse, at every scale sub-sampling, than the framework using $\mathrm{PC}$ edge map (MSDEPC) implies that, for the SISR task, incorporating more types of image features actually cannot guarantee a better reconstruction performance. It is clear that MSDEPC and MSDEPC-V1 both integrating PC edge maps, demonstrate much stronger performance than others. This observation might indicate that PC edge map is indeed a kind of effective edge features for improving SISR.

Moreover, based on the performance comparisons between MSDEPC$\mathrm{V} 1$ and MSDEPC in the table, it is also clear that MSDEPC is supeior to MSDEPC-V1. This result strongly suggests us that multi-scale framework is more favorable for SISR compared to single scale structure. Convergence Acceleration with $B N$ and $G C$. In Table 3, we compare the convergence speed of the proposed models with and without the techniques of $\mathrm{BN}$ and $\mathrm{GC}$ by trained epochs measure. It is clear that with batch normalization and gradient clipping, the network may require less training epochs to achieve convergence. 
Table 3: Convergence speed comparisons: with or without BN and GC.

\begin{tabular}{ccccccccc}
\hline \multirow{2}{*}{ Training epochs } & \multicolumn{2}{c}{ MSDEPC } & \multicolumn{2}{c}{ MSDEPC-V1 } & \multicolumn{2}{c}{ MSDEPC-V2 } & \multicolumn{2}{c}{ MSDEPC-V3 } \\
\cline { 2 - 9 } & $\times 2$ & $\times 4$ & $\times 2$ & $\times 4$ & $\times 2$ & $\times 4$ & $\times 2$ & $\times 4$ \\
With BN and GC & 913 & 85 & 796 & 75 & 131 & 62 & 527 & 160 \\
W/O BN and GC & 1097 & 167 & 821 & 143 & 201 & 75 & 626 & 234 \\
\hline
\end{tabular}

Multiple Scales Edge Prediction. To show the ability of the proposed multiscale model, the predicted three scales' edge maps of two test images during a training session are shown in Fig. 8. From the figure, it is clear that training the model can capture image edge details at different scales. The shortest encoder-decoder stream (4 layers), which corresponds to coarse scale, can capture all massive edges; the middle length encoder-decoder stream (8 layers), corresponding to the medium scale, can strengthen the meso-scale edges; while the longest cascading one (12 layers), corresponding to the fine scale, can acquire the smallest edge details. Additionally, it is also shown the more layers involved in the network, the finer the scale will be.
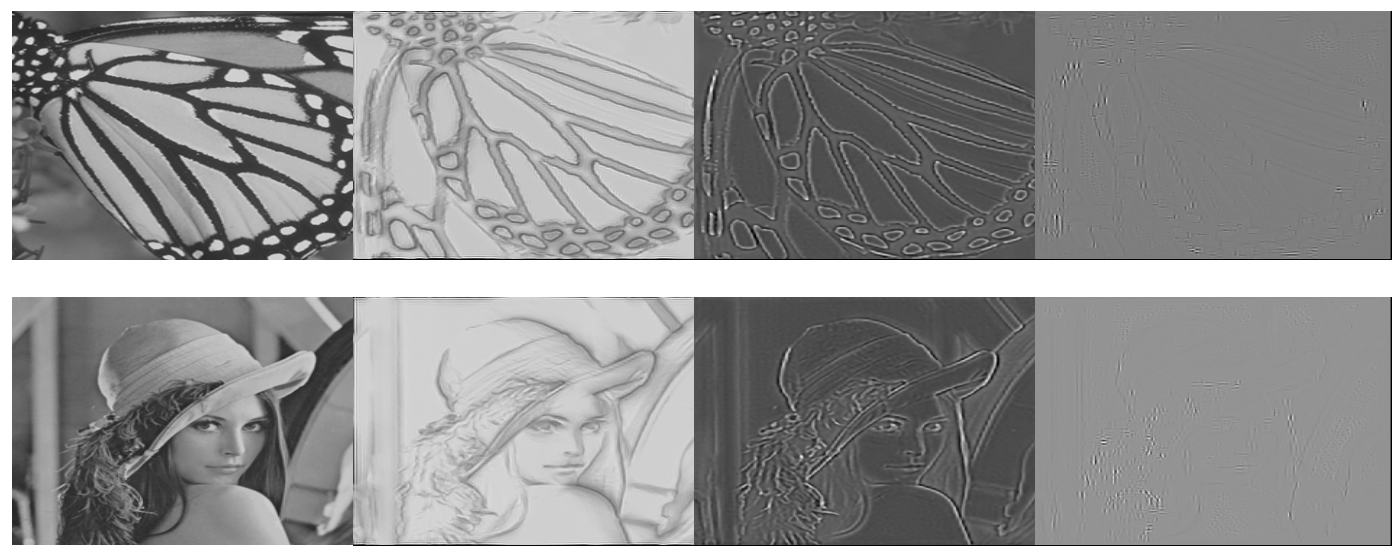

(a) Input image

(b) Corase scale edge (c) Medium scale (d) Fine scale edge prediction edge prediction prediction

Figure 8: Multi-scale edge prediction extracted from a network training session. From left to right: original image, coarse scale (4 layers) edge prediction, medium scale (8 layers) edge prediction and fine scale (12 layers) one.

Comparisons to the State-of-the-Art. Here, we compare the proposed method with other recently published state-of-the-art methods, including SRCNN-L [5], ESPCN [33], SelfExSR [11] and VDSR [15].We evaluate our methods in terms of widely used measures PSNR and SSIM [36]. Here SRCNN refers to its 9-1-5 model while SRCNN-L denotes the longer 9-5-5 ImageNet one. Since the works $[14,18]$ associate the loss of SR with the feature maps of VGG network and argue that PSNR and SSIM are not the appropriate metrics for 
SISR, for fairness, we do not make comparisons with them. Depending on the training datasets, the comparisons are divided into two groups: Table 4 lists the methods trained by 91-images while Table 5 shows those trained by ImageNet or others. According to the results in Table 4 and Table 5, the proposed MSDEPC model can obtain the best performance in most cases, and only performs a little worse at several particular spots than the VDSR [15], which is the best published algorithm. From the algorithm perspective, the reason why VDSR is occasionally better than us might be the deeper network (20 vs 12 ) with more filters in each layer (64 vs 32 ), which inspires us to deepen our multi-scale architecture and make more dense filters in future work.

The running time comparisons (three times average) of $4 \times$ SISR for $512 \times 512$ input between the proposed model and the other CNN-based methods are shown in Table 6. From the table, it is clear that if using GPU to perform SISR, the time-consuming difference between the proposed method and the other methods is almost smoothed out. But the gap does exist if CPU is used. At such time, the proposed method is about five times faster than VDSR and only a little slower than SRCNN-L (only contains three layers). The visual qualities of the super-resolved images generated by our model and also the other competing models based on different test datasets are illustrated in Fig. 9 and Fig. 10.

Table 4: The mean PSNR (dB) (left numbers) and SSIM (right numbers) for different methods trained with 91-images. Best results are indicated in Bold.

\begin{tabular}{|c|c|c|c|c|c|c|c|c|c|}
\hline \multirow{2}{*}{ Dataset } & \multicolumn{3}{|c|}{ Set5 } & \multicolumn{3}{|c|}{ Set14 } & \multicolumn{3}{|c|}{ BSD100 } \\
\hline & $\times 2$ & $\times 3$ & $\times 4$ & $\times 2$ & $\times 3$ & $\times 4$ & $\times 2$ & $\times 3$ & $\times 4$ \\
\hline Bicubic & $33.66 \backslash 0.9299$ & $30.39 \backslash 0.8682$ & $28.42 \backslash 0.8104$ & $30.24 \backslash 0.8687$ & $27.55 \backslash 0.7736$ & $26.00 \backslash 0.7019$ & $29.56 \backslash 0.8431$ & $27.21 \backslash 0.7385$ & $25.96 \backslash 0.6675$ \\
\hline $\operatorname{SRCNN}[4]$ & $36.34 \backslash 0.9521$ & $32.37 \backslash 0.9033$ & $30.03 \backslash 0.8530$ & $32.18 \backslash 0.9017$ & $29.00 \backslash 0.8145$ & $27.20 \backslash 0.7413$ & $31.11 \backslash 0.8835$ & $28.20 \backslash 0.7794$ & $26.70 \backslash 0.7078$ \\
\hline ESPCN[33] & $-\backslash-$ & $32.55 \backslash-$ & $-\backslash-$ & $-\backslash-$ & $29.08 \backslash-$ & $-\backslash-$ & $-\backslash-$ & $28.26 \backslash-$ & $-\backslash-$ \\
\hline MSDEPC & $37.39 \backslash 0.9576$ & $33.37 \backslash 0.9184$ & $31.05 \backslash 0.8797$ & $32.94 \backslash 0.9111$ & $29.62 \backslash 0.8279$ & $27.79 \backslash 0.7581$ & $31.64 \backslash 0.8961$ & $28.58 \backslash 0.7918$ & $27.10 \backslash 0.7193$ \\
\hline
\end{tabular}

In addition, we also apply the proposed model for different types of images, such as depth images, in order to validate its generalizability. Here the depth data is taken from the Middlebury dataset [31] and the New Tsukuba dataset [26]. It should be noted that for these super-resolution experiments, we just take the model trained from 91-images and do not make any further fine-tuning. The visual results and the comparisons (Fig. 11) indicate the wide applicability of our proposed model. 


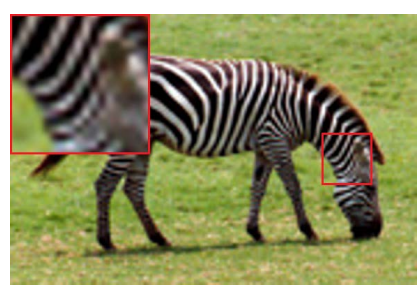

(a) Bicubic: 24.08

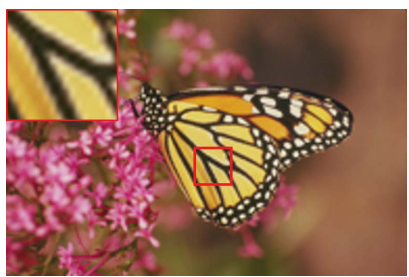

(a) Bicubic: 27.55

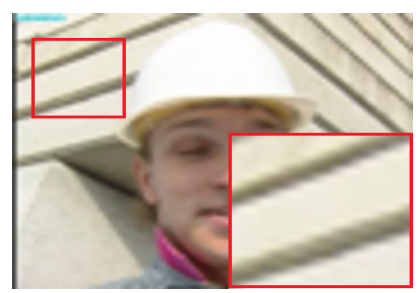

(a) Bicubic: 29.41

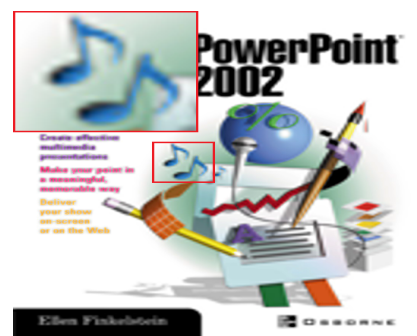

(a) Bicubic: 21.98

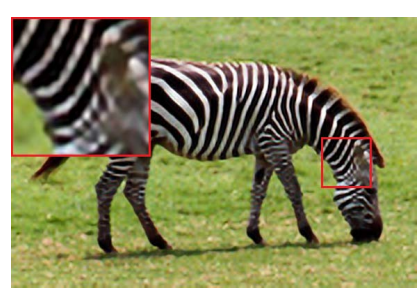

(b) SRCNN-L: 26.09

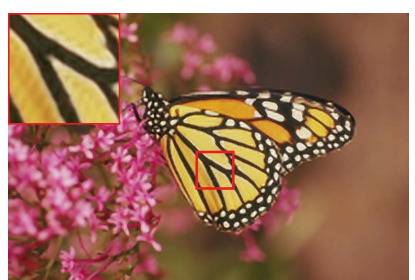

(b) SRCNN-L: 30.22

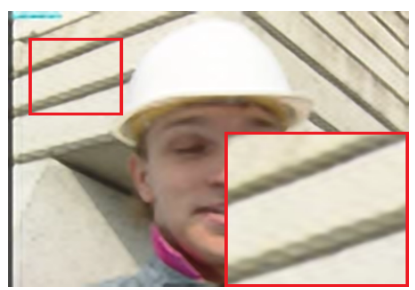

(b) SRCNN-L: 32.24

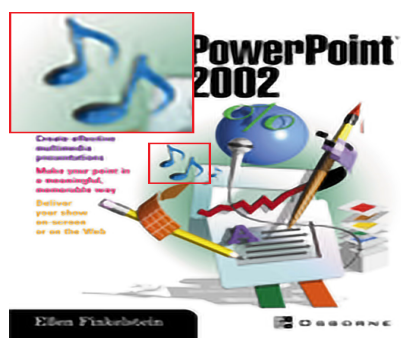

(b) SRCNN-L: 24.80

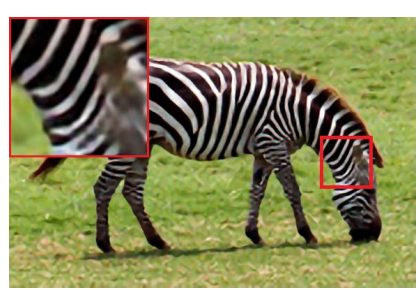

(c) MSDEPC: 26.54

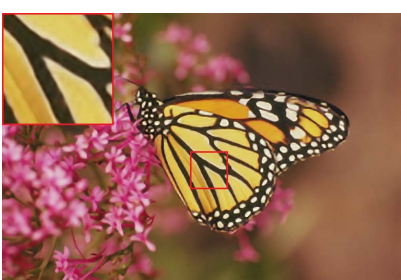

(c) MSDEPC: 30.83

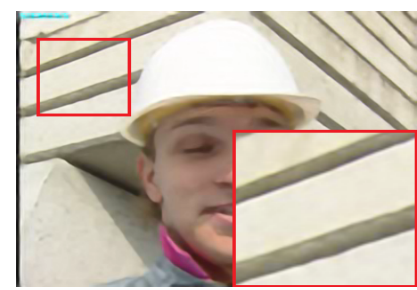

(c) MSDEPC: 32.90

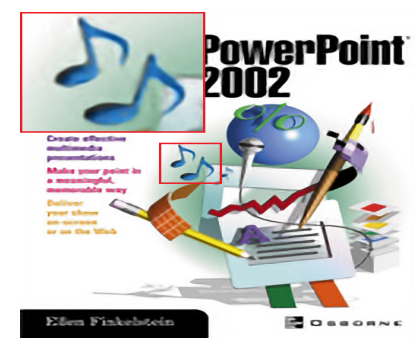

(c) MSDEPC: 25.78

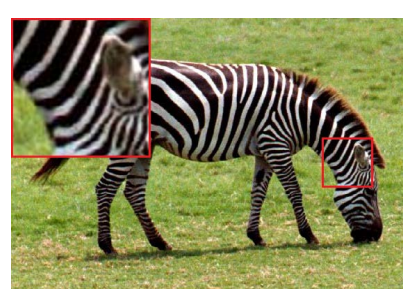

(d) Original: Zebra

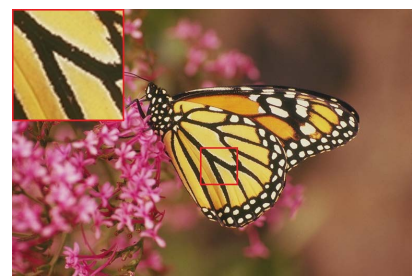

(d) Orignal: Monarch

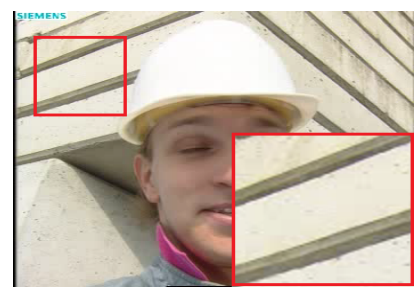

(d) Orignal: Monarch

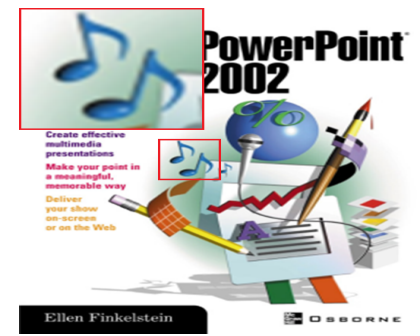

(d) Orignal: Monarch

Figure 9: Visual and PSNR (db) comparisons of super-resolved $(4 \times)$ images for 'Zebra', 'Monarch', 'Fireman', and 'ppt3' from Set14 by (a) Bicubic, (b) SRCNN-L, and (c) the proposed - MSDEPC, respectively. 


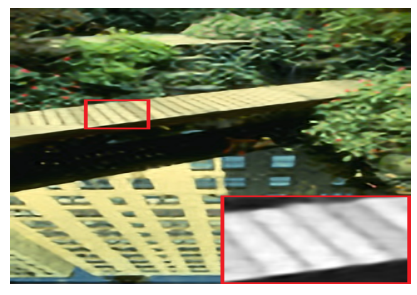

(a) SRCNN-L: 21.53

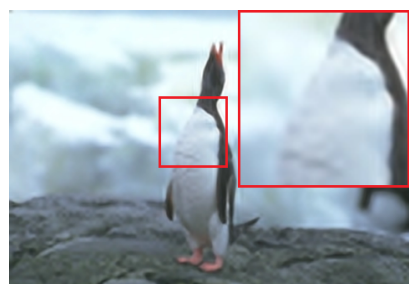

(a) SRCNN-L: 33.01

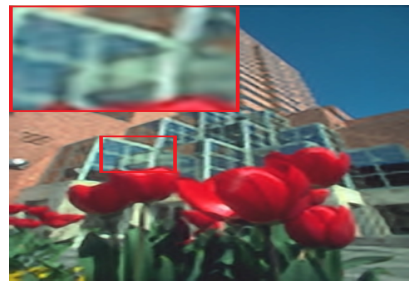

(a) SRCNN-L: 24.80

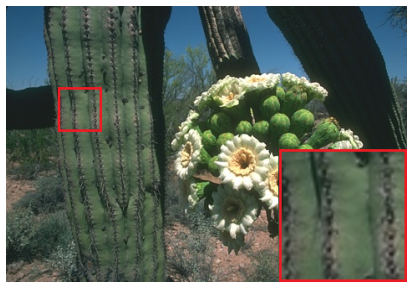

(a) SRCNN-L: $26.36 \mathrm{db}$

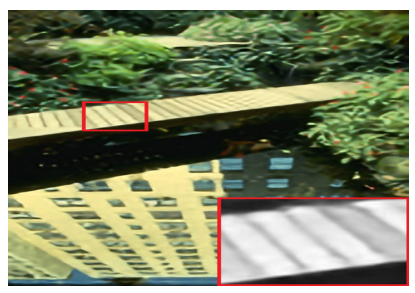

(b) VDSR: 21.71

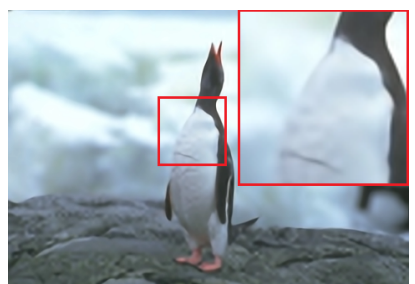

(b) VDSR: 33.67

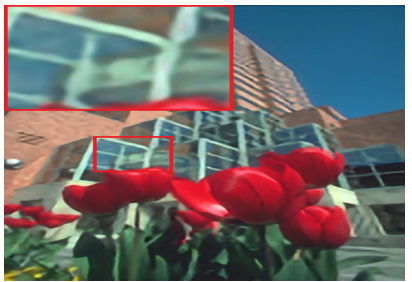

(b) VDSR: 25.85

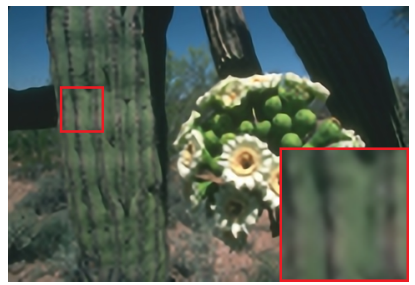

(b) VDSR: $26.61 \mathrm{db}$

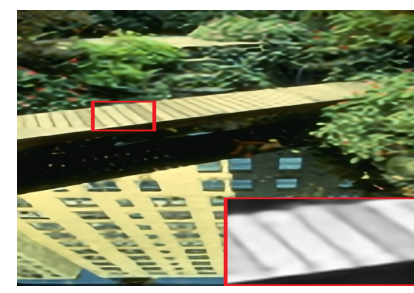

(c) MSDEPC: 21.82

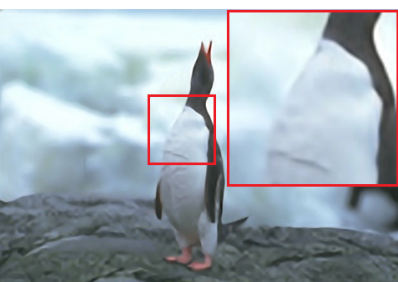

(c) MSDEPC: 33.78

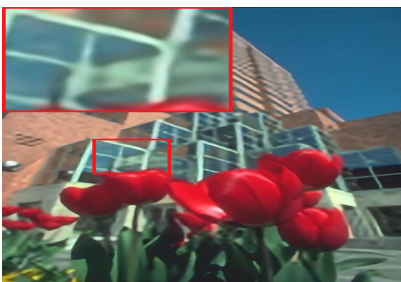

(c) MSDEPC: 25.93

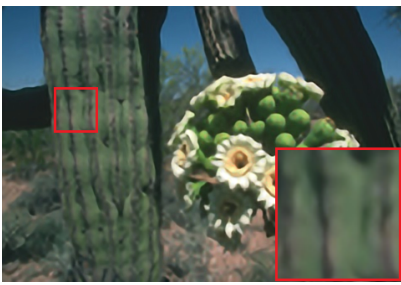

(c) MSDEPC: $26.67 \mathrm{db}$

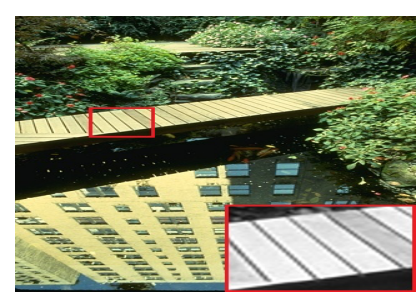

(d) Original: 148026

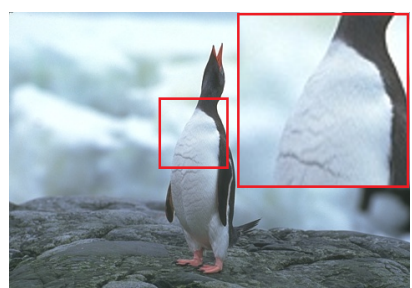

(d) Orignal: 106024

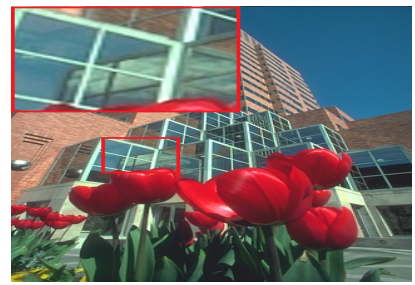

(d) Original: 86000

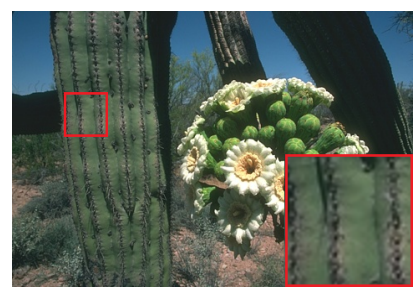

(d) Orignal: 19201

Figure 10: Visual and PSNR (db) comparisons of super-resolved $(4 \times)$ images for '140826', '106024', '86000', and '19021' from BSD100 by (a) SRCNN-L, (b) VDSR, and (c) the proposed - MSDEPC, respectively. 


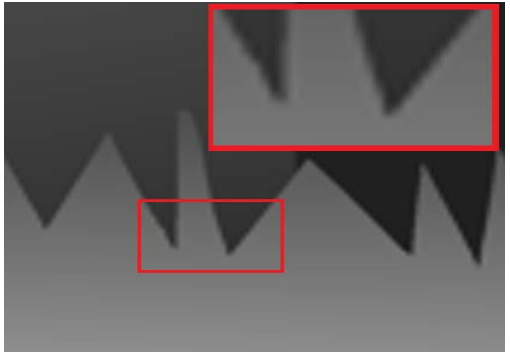

(a) Bicubic: $37.43 \mathrm{db}$

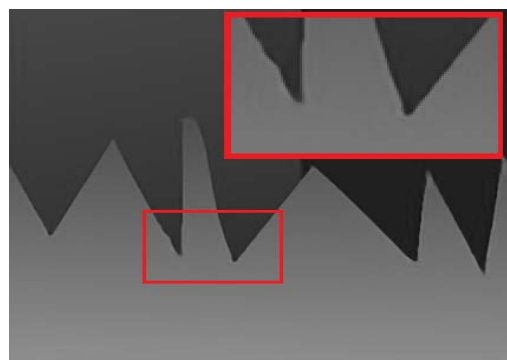

(c) MSDEPC: $41.67 \mathrm{db}$

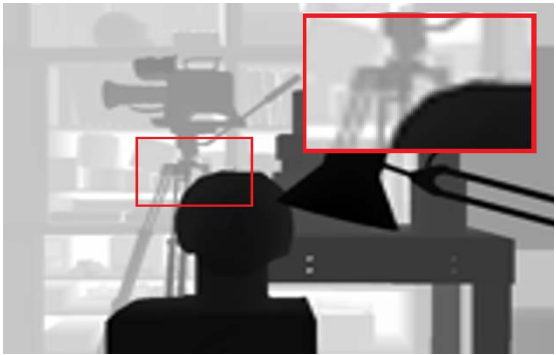

(a) Bicubic: $27.43 \mathrm{db}$

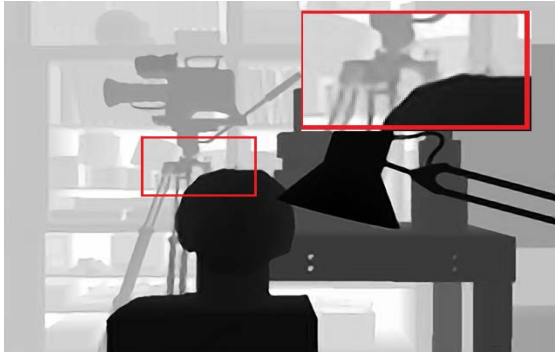

(c) MSDEPC: $31.59 \mathrm{db}$

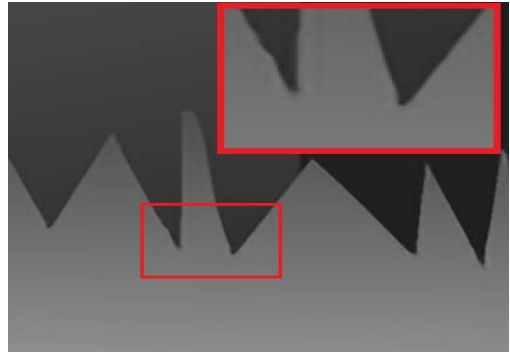

(b) SRCNN-L: $40.84 \mathrm{db}$

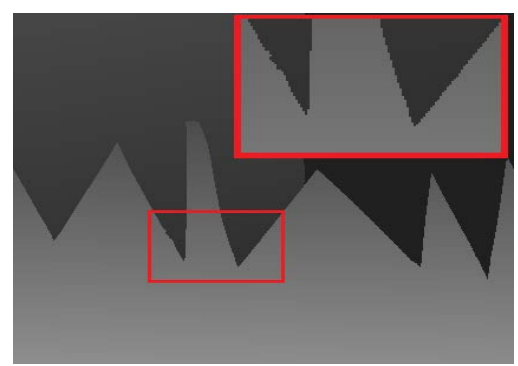

(d) Orignal: Middlebury

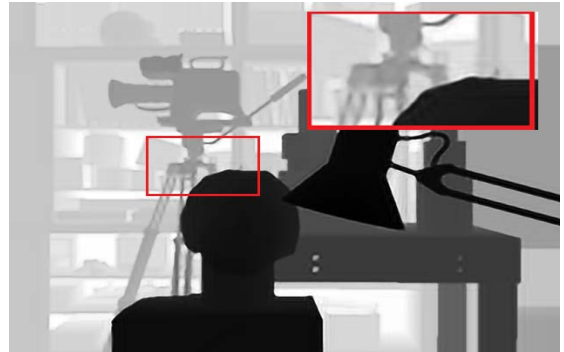

(b) SRCNN-L: $30.84 \mathrm{db}$

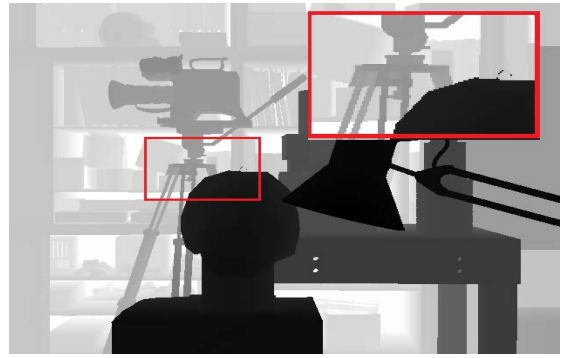

(d) Original: New Tsukuba

Figure 11: Super-resolved $(4 \times)$ images for depth data from Middlebury [31] (upper part) and New Tsukuba [26] (lower part) by (a) Bicubic, (b) SRCNN-L, and (c) the proposed MSDEPC, respectively. 
Table 5: The mean PSNR (dB) (left numbers) and SSIM (right numbers) for different methods trained with ImageNet or others. Best results are indicated in Bold.

\begin{tabular}{ccccccccccc}
\hline \multirow{2}{*}{ Dataset } & \multicolumn{3}{c}{ Set5 } & \multicolumn{3}{c}{ Set14 } & & BSD100 & \\
\cline { 2 - 10 } & $\times 2$ & $\times 3$ & $\times 4$ & $\times 2$ & $\times 3$ & $\times 4$ & $\times 2$ & $\times 3$ & $\times 4$ \\
SRCNN-L[5] & $36.66 \backslash 0.9542$ & $32.75 \backslash 0.9090$ & $30.49 \backslash 0.8628$ & $32.45 \backslash 0.9027$ & $29.30 \backslash 0.8251$ & $27.50 \backslash 0.7513$ & $31.36 \backslash 0.8876$ & $28.41 \backslash 0.7853$ & $26.90 \backslash 0.7186$ \\
SelfExSR[11] & $36.62 \backslash 0.9548$ & $32.66 \backslash 0.9098$ & $30.35 \backslash 0.8607$ & $32.31 \backslash 0.9070$ & $29.16 \backslash 0.8209$ & $27.30 \backslash 0.7499$ & $31.32 \backslash 0.8835$ & $28.33 \backslash 0.7778$ & $26.80 \backslash 0.7120$ \\
ESPCN[33] & $-\backslash-$ & $33.13 \backslash-$ & $30.90 \backslash-$ & $-\backslash-$ & $29.49 \backslash-$ & $27.73 \backslash-$ & $-\backslash-$ & $28.54 \backslash-$ & $27.06 \backslash-$ \\
VDSR[15] & $37.53 \backslash 0.9578$ & $33.65 \backslash 0.9210$ & $31.33 \backslash 0.8834$ & $\mathbf{3 3 . 0 3} \backslash \mathbf{0 . 9 1 2 4}$ & $29.75 \backslash 0.8312$ & $27.95 \backslash 0.7671$ & $31.90 \backslash 0.8960$ & $28.80 \backslash 0.7970$ & $27.24 \backslash 0.7245$ \\
MSDEPC & $\mathbf{3 7 . 5 4} \backslash \mathbf{0 . 9 5 8 7}$ & $\mathbf{3 3 . 7 0} \backslash \mathbf{0 . 9 2 2 5}$ & $\mathbf{3 1 . 4 1} \backslash \mathbf{0 . 8 8 3 6}$ & $32.96 \backslash 0.9117$ & $\mathbf{2 9 . 7 8} \backslash \mathbf{0 . 8 3 1 9}$ & $\mathbf{2 8 . 0 2} \backslash \mathbf{0 . 7 6 7 9}$ & $\mathbf{3 1 . 9 2} \backslash \mathbf{0 . 8 9 6 7}$ & $\mathbf{2 8 . 8 8} \backslash \mathbf{0 . 7 9 7 4}$ & $\mathbf{2 7 . 3 0} \backslash \mathbf{0 . 7 2 4 9}$ \\
\hline
\end{tabular}

Table 6: Running time comparisons (Seconds; with one TITAN X).

\begin{tabular}{cccc}
\hline Running time & SRCNN-L & VDSR & MSDEPC \\
With GPU & 0.188 & 0.245 & 0.241 \\
Without GPU & 11.417 & 74.053 & 15.269 \\
\hline
\end{tabular}

\section{Conclusion}

In this work, by presenting a new MSDEPC model, we have demonstrated that multi-scale deep structure with appropriate edge details integration will significantly facilitate the task of SISR.

We explored the relationship between signal wavelet multi-scale analysis and multi-scale encoder-decoder learning networks, based on which we have constructed a three-scale encoder-decoder deep model for SISR. Moreover, we integrated the important image structural features - phase congruency edge into the multi-scale network to ensure the recovery of image structural edge details. Experimental comparisons showed that the proposed approach outperforms the state-of-the-art methods.

Future work will focus on two aspects. Directly learning upscaling-filters by introducing the deconvolution into each scale network is the near future task. Investigating the perception loss and fusing image classification task with super-resolution into current multi-scale learning architecture will be the long term target.

\section{Acknowledgment}

This work is partly supported by the Major Project and Key Project of Natural Science of Anhui Provincial Department of Education (Grant No. KJ2015ZD09 and KJ2016A085). It is also supported by Anhui Provincial Natural Science Foundation (Grant No. 1608085MF129). 


\section{References}

[1] Bergstra, J., Bengio, Y., 2012. Random search for hyper-parameter optimization. Journal of Machine Learning Research 13 (Feb), 281-305.

[2] Bevilacqua, M., Roumy, A., Guillemot, C., Alberi-Morel, M. L., 2012. Low-complexity single-image super-resolution based on nonnegative neighbor embedding. In: Proc. British Machine Vision Conf. pp. 1-10.

[3] Choi, J.-S., Kim, M., 2017. Single image super-resolution using global regression based on multiple local linear mappings. IEEE Transactions on Image Processing 26 (3), 1300-1314.

[4] Dong, C., Loy, C. C., He, K., Tang, X., 2014. Learning a deep convolutional network for image super-resolution. In: Proc. Eur. Conf. Comput. Vis. pp. 184-199.

[5] Dong, C., Loy, C. C., He, K., Tang, X., 2016. Image super-resolution using deep convolutional networks. IEEE Trans. Pattern Anal. Mach. Intell. 38 (2), 295-307.

[6] Gu, S., Zuo, W., Xie, Q., Meng, D., Feng, X., Zhang, L., 2015. Convolutional sparse coding for image super-resolution. In: Proc. IEEE Int. Conf. Comput. Vis. Pattern Recognit. pp. 1823-1831.

[7] Han, W., Chang, S., Liu, D., Yu, M., Witbrock, M., Huang, T. S., 2018. Image super-resolution via dual-state recurrent networks. In: Proceedings of the IEEE Conference on Computer Vision and Pattern Recognition. pp. 1654-1663.

[8] Haris, M., Shakhnarovich, G., Ukita, N., 2018. Deep back-projection networks for super-resolution. In: Proceedings of the IEEE Conference on Computer Vision and Pattern Recognition. pp. 1664-1673.

[9] He, K., Zhang, X., Ren, S., Sun, J., 2015. Deep residual learning for image recognition. arXiv preprint arXiv:1512.03385.

[10] He, K., Zhang, X., Ren, S., Sun, J., 2015. Delving deep into rectifiers: Surpassing human-level performance on imagenet classification. In: Proc. IEEE Int. Conf. Comput. Vis. Pattern Recognit. pp. 10261034. 
[11] Huang, J.-B., Singh, A., Ahuja, N., 2015. Single image super-resolution from transformed self-exemplars. In: Proc. IEEE Int. Conf. Comput. Vis. Pattern Recognit. pp. 5197-5206.

[12] Ioffe, S., Szegedy, C., 2015. Batch normalization: Accelerating deep network training by reducing internal covariate shift. arXiv preprint arXiv:1502.03167.

[13] Jia, Y., Shelhamer, E., Donahue, J., Karayev, S., Long, J., Girshick, R., Guadarrama, S., Darrell, T., 2014. Caffe: Convolutional architecture for fast feature embedding. In: Proc. ACM Int. Conf. Multimedia. pp. 675-678.

[14] Johnson, J., Alahi, A., Fei-Fei, L., 2016. Perceptual losses for real-time style transfer and super-resolution. In: Proc. Eur. Conf. Comput. Vis. pp. 694-711.

[15] Kim, J., Kwon Lee, J., Mu Lee, K., 2016. Accurate image superresolution using very deep convolutional networks. In: Proc. IEEE Int. Conf. Comput. Vis. Pattern Recognit. pp. 1646-1654.

[16] Kovesi, P., 1999. Image features from phase congruency. J. Comput. Vis. Res. 1 (3), 1-26.

[17] Kovesi, P., 2003. Phase congruency detects corners and edges. In: Proc. Australian Pattern Recog. Soc. Conf.

[18] Ledig, C., Theis, L., Huszár, F., Caballero, J., Aitken, A., Tejani, A., Totz, J., Wang, Z., Shi, W., 2016. Photo-realistic single image super-resolution using a generative adversarial network. arXiv preprint arXiv:1609.04802.

[19] Li, Z., Tang, J., 2015. Weakly supervised deep metric learning for community-contributed image retrieval. IEEE Transactions on Multimedia 17 (11), 1989-1999.

[20] Li, Z., Tang, J., 2017. Weakly supervised deep matrix factorization for social image understanding. IEEE Transactions on Image Processing 26 (1), 276-288. 
[21] Liang, Y., Wang, J., Zhou, S., Gong, Y., Zheng, N., 2016. Incorporating image priors with deep convolutional neural networks for image superresolution. Neurocomputing 194, 340-347.

[22] Long, J., Shelhamer, E., Darrell, T., 2015. Fully convolutional networks for semantic segmentation. In: Proc. IEEE Int. Conf. Comput. Vis. Pattern Recognit. pp. 3431-3440.

[23] Ma, C., Yang, C.-Y., Yang, X., Yang, M.-H., 2017. Learning a noreference quality metric for single-image super-resolution. Computer Vision and Image Understanding 158, 1-16.

[24] Mallat, S., 1999. A wavelet tour of signal processing. Academic press.

[25] Martin, D., Fowlkes, C., Tal, D., Malik, J., 2001. A database of human segmented natural images and its application to evaluating segmentation algorithms and measuring ecological statistics. In: Proc. IEEE Int. Conf. Comput. Vis. Vol. 2. pp. 416-423.

[26] Martull, S., Peris, M., Fukui, K., 2012. Realistic cg stereo image dataset with ground truth disparity maps. In: ICPR workshop TrakMark2012. Vol. 111. pp. 117-118.

[27] Odena, A., Dumoulin, V., Olah, C., 2016. Deconvolution and checkerboard artifacts. http://distill.pub/2016/deconv-checkerboard/.

[28] Pan, J., Liu, S., Sun, D., Zhang, J., Liu, Y., Ren, J., Li, Z., Tang, J., Lu, H., Tai, Y.-W., et al., 2018. Learning dual convolutional neural networks for low-level vision. In: Proceedings of the IEEE Conference on Computer Vision and Pattern Recognition. pp. 3070-3079.

[29] Ren, W., Liu, S., Zhang, H., Pan, J., Cao, X., Yang, M.-H., 2016. Single image dehazing via multi-scale convolutional neural networks. In: Proc. Eur. Conf. Comput. Vis. pp. 154-169.

[30] Russakovsky, O., Deng, J., Su, H., Krause, J., Satheesh, S., Ma, S., Huang, Z., Karpathy, A., Khosla, A., Bernstein, M., et al., 2015. Imagenet large scale visual recognition challenge. Int. J. Comput. Vision 115 (3), 211-252. 
[31] Scharstein, D., Szeliski, R., 2002. A taxonomy and evaluation of dense two-frame stereo correspondence algorithms. Int. J. Comput. Vision $47(1-3), 7-42$.

[32] Shen, W., Zhao, K., Jiang, Y., Wang, Y., Zhang, Z., Bai, X., 2016. Object skeleton extraction in natural images by fusing scale-associated deep side outputs. arXiv preprint arXiv:1603.09446.

[33] Shi, W., Caballero, J., Huszár, F., Totz, J., Aitken, A. P., Bishop, R., Rueckert, D., Wang, Z., 2016. Real-time single image and video superresolution using an efficient sub-pixel convolutional neural network. In: Proc. IEEE Int. Conf. Comput. Vis. Pattern Recognit. pp. 1874-1883.

[34] Simonyan, K., Zisserman, A., 2014. Very deep convolutional networks for large-scale image recognition. arXiv preprint arXiv:1409.1556.

[35] Timofte, R., De Smet, V., Van Gool, L., 2014. A+: Adjusted anchored neighborhood regression for fast super-resolution. In: Proc. IEEE Asian Conf. Comput. Vis. pp. 111-126.

[36] Wang, Z., Bovik, A. C., Sheikh, H. R., Simoncelli, E. P., 2004. Image quality assessment: from error visibility to structural similarity. IEEE Trans. Image Process. 13 (4), 600-612.

[37] Wang, Z., Liu, D., Yang, J., Han, W., Huang, T., 2015. Deep networks for image super-resolution with sparse prior. In: Proc. IEEE Int. Conf. Comput. Vis. Pattern Recognit. pp. 370-378.

[38] Xie, J., Feris, R. S., Sun, M.-T., 2016. Edge-guided single depth image super resolution. IEEE Trans. Image Process. 25 (1), 428-438.

[39] Xie, S., Tu, Z., 2015. Holistically-nested edge detection. In: Proc. IEEE Int. Conf. Comput. Vis. pp. 1395-1403.

[40] Yang, J., Price, B., Cohen, S., Lee, H., Yang, M.-H., 2016. Object contour detection with a fully convolutional encoder-decoder network. arXiv preprint arXiv:1603.04530.

[41] Yang, J., Wang, Z., Lin, Z., Cohen, S., Huang, T., 2012. Coupled dictionary training for image super-resolution. IEEE Trans. Image Process. 21 (8), 3467-3478. 
[42] Yang, J., Wright, J., Huang, T. S., Ma, Y., 2010. Image super-resolution via sparse representation. IEEE Trans. Image Process. 19 (11), 28612873.

[43] Yang, W., Feng, J., Yang, J., Zhao, F., Liu, J., Guo, Z., Yan, S., 2016. Deep edge guided recurrent residual learning for image super-resolution. arXiv preprint arXiv:1604.08671.

[44] Zeiler, M. D., Fergus, R., 2014. Visualizing and understanding convolutional networks. In: Proc. Eur. Conf. Comput. Vis. pp. 818-833.

[45] Zeyde, R., Elad, M., Protter, M., 2010. On single image scale-up using sparse-representations. In: Proc. Int. Conf. Curves Surf. pp. 711-730.

[46] Zhang, K., Wang, B., Zuo, W., Zhang, H., Zhang, L., 2016. Joint learning of multiple regressors for single image super-resolution. IEEE Signal. Proc. Let. 23 (1), 102-106.

[47] Zhang, Z., Jiang, W., Li, F., Zhao, M., Li, B., Zhang, L., 2017. Structured latent label consistent dictionary learning for salient machine faults representation-based robust classification. IEEE Transactions on Industrial Informatics 13 (2), 644-656.

[48] Zhang, Z., Li, F., Zhao, M., Zhang, L., Yan, S., 2017. Robust neighborhood preserving projection by nuclear/12, 1-norm regularization for image feature extraction. IEEE Transactions on Image Processing 26 (4), $1607-1622$.

[49] Zhu, Y., Zhang, Y., Yuille, A. L., 2014. Single image super-resolution using deformable patches. In: Proc. IEEE Int. Conf. Comput. Vis. Pattern Recognit. pp. 2917-2924. 